\title{
Absolute proper motions for the calibration of the Hipparcos proper motion system
}

\author{
M. Geffert ${ }^{1}$, A.R. Klemola ${ }^{2}$, M. Hiesgen ${ }^{3}$, and J. Schmoll ${ }^{1}$ \\ 1 Sternwarte der Universität Bonn, Auf dem Hügel 71, D-53121 Bonn, Germany \\ 2 Lick Observatory, University of California, UCSC, Santa Cruz CA 95064, U.S.A. \\ 3 Astronomisches Institut der Universität Münster, Wilhelm-Klemm-Str. 10 D-48149 Münster, Germany
}

Received September 11; accepted November 20, 1996

\begin{abstract}
Absolute proper motions of 48 Hipparcos stars in nine fields distributed over the northern hemisphere are presented. The proper motions have been recently used as part of the Bonn program for the extragalactic link of the Hipparcos proper motion system. We describe methods which have led to the absolute proper motions of the major part of the Bonn fields for the link. For six fields the extragalactic calibration was achieved using the bright quasar in the centre of the field. In addition, data of three fields of globular clusters were included. For these fields the link to an extragalactic reference system was performed by stars, whose absolute proper motions with respect to galaxies were determined on plates of the Lick astrograph. In addition, we used for the link in one field plates from the ESO Schmidt telescope in combination with measurements from glass copies of the Palomar Sky Survey. The accuracy of a single proper motion is of the order of 2 to 3 mas/a for each coordinate. ${ }^{1}$
\end{abstract}

Key words: astrometry — reference system — stars: kinematics

\section{Introduction}

The forthcoming proper motions of more than 100.000 stars provided by Hipparcos with an accuracy of better than 2 mas/a will be an important tool for the study of stellar kinematics and dynamics. However the Hipparcos satellite was only able to provide relative proper motion data. Therefore the proper motions of the Hipparcos satellite had to be calibrated with other techniques (e.g. Froeschlé \& Kovalevsky 1982). As already proposed by

Send offprint requests to: M. Geffert

1 Table 2 is also available in electronic form at the CDS via anonymous ftp to cdsarc.u-strasbg.fr (130.79.128.5) or via http://cdsweb.u-strasbg.fr/Abstract.html
Brosche (1980) one promising method for the calibration of absolute proper motions is the use of extragalactic objects on photographic plates. While first results for the calibration of proper motions were already published (e.g. Brosche \& Geffert 1981; Brosche et al. 1991a; Geffert et al. 1993), we present here absolute proper motions of 48 stars, which were also measured by the Hipparcos satellite. Our data have been recently used for a complete Hipparcos link solution of all Bonn fields (Tucholke et al. 1997) and for the final link of the Hipparcos proper motion system (Kovalevsky et al. 1997).

\section{Searching for suited fields and first epoch material}

In order to obtain accurate proper motions (better than 3 mas/a for a single star) it was clear that only first epoch plates from earlier than 1940 from telescopes with focal lengths larger than $2 \mathrm{~m}$ could be used. Another constraint for the first epoch material was that the available field should cover not less than $1.5^{\circ} \times 1.5^{\circ}$. The use of such old material implies that only fields with bright $(V<16)$ extragalactic objects were suited. In the beginning we also restricted our search mainly to fields with (optical) pointlike objects, for which also an accurate radio position from VLBI was available. Our search for bright candidates resulted in a first list of candidates, which was published by Brosche (1980). In the following years we have made an extensive search for first epoch plates in different plate vaults mainly in Europe starting with the plate vault of our double refractor of Bonn (now located at Hoher List observatory). Especially the quoted brightness of the objects turned out to be quite uncertain. One of the reasons is the strong optical variability of some of the objects. First epoch plates of about fifteen candidates were detected and partly measured, but only for six objects the material seemed promising for a complete measurement and reduction. A list of all link fields used in this project is given in Table 1. Moreover, plates of the optical extended 
objects M 51 and M 94 were found in our own archive, the M 51 data were later analysed with additional observational data and a refined method of measurement and reduction (Odenkirchen \& Brosche 1995).

In addition to the fields with one extragalactic object we used data from the Bonn globular cluster program for the determination of absolute proper and space motions of globular clusters (e.g. Geffert 1987; Brosche et al. 1991b; Geffert et al. 1993).

\section{Second epoch observations, measurements and reductions}

New observations for the complete Bonn link were mainly taken at the double refractor $(D=0.3 \mathrm{~m}, f=5.1 \mathrm{~m})$ from 1978 to 1994. 103 a-O, IIa-O and IIIa-J emulsions have been used with a BG3 or BG25 filter. Part of these plates has been used by others (see Tucholke et al. 1997 and references therein) for additional link fields. In addition to the observations of our own refractor, we have used also plates of the $2 \mathrm{~m} \mathrm{RC}$ telescope at Rozhen observatory and the $1.23 \mathrm{~m}$ telescope at Calar Alto, mainly for those fields, for which the objects were too faint for our double refractor. Data of the plates and telescopes for the complete observations may be found in Geffert et al. (1989), Brosche et al. (1991a) and Dick et al. (1993).

Measurements were performed either by scanning the complete plate at the PDS $2020 \mathrm{GM}^{\text {plus }}$ in Münster or by measuring about 100 stars equally distributed over the plate on the ASCORECORD of the Bonner Sternwarte. In the latter case each plate was measured in two opposite directions in order to avoid systematic errors of the measurements due to drift of the machine or personal magnitude equation.

For the reduction, to achieve a well defined local coordinate grid, a complete set of FORTRAN routines was established. The method of reduction includes variable plate constant models with polynomials of the rectangular coordinates $x$ and $y$, colours and magnitudes. However the colour of our objects was generally unknown and not included in the reduction. An iterative central plate overlap algorithm was used. A more detailed description of the reduction procedure is given in LeCampion et al. (1992). The complete results of the Bonn part of the Hipparcos link (Tucholke et al. 1997) were based on these routines or modifications of them. In the beginning, the reduction was performed with the PPM catalogue. Later a study of the proper motions of the Pleiades cluster (Geffert et al. 1995) had shown that even using the central plate overlap algorithm the results depend in a strong way on the reference stars used at the beginning of the iteration. Therefore we used the Hipparcos catalogue for the 37 month solution of Hipparcos (H37) in fields with enough reference stars for each plate.

The use of this catalogue was the most promising method to avoid systematic errors in our proper motions by imperfect reference stars. Nevertheless we wish to point out, that the final proper motions determined by an iterative plate reduction procedure are mainly based on our plate material. Moreover, for our final proper motions in Table 2, only the link procedure described in Sect. 4 determines for each field separately the system of the proper motions, which is completely independent of the H37.

However, the number of reference stars in our reduction varies from only three to nine. For the field with nine Hipparcos stars (NGC 4147), we performed different independent reductions using only part of the available reference stars and compared the different results of our reduction. The comparison demonstrated the stability of the reduction using the $\mathrm{H} 37$ reference stars. The mean differences of the proper motions of the final solutions are below $1 \mathrm{mas} / \mathrm{a}$. This result is of great importance for all future work on the Bonn plates, because these fields normally contain only 4 to 6 Hipparcos stars.

\section{Extragalactic calibration of the proper motions}

A very important step for getting absolute proper motions is the extragalactic calibration of the proper motions. We have used three different kind of methods. The first method is based on one bright extragalactic object in the centre of our field. Since we don't expect to measure any real motion of such distant objects, we may assume that the measured proper motion represents only a zero point error of our system. Therefore we have to subtract the apparent proper motions of the extragalactic object from all proper motions for the calibration. This method is denoted with EO in Col. 5 of Table 1. The internal errors of our proper motions give a lower limit to the accuracy of the calibration of our proper motions. In each coordinate we obtained 1 to 2 mas/a for our objects. The drawback of this method is, that the use of only one object for the calibration does not allow a correction for a possible rotation etc. of the proper motions in the field. Unfortunately none of our plates contains more than one bright extragalactic object, which would improve the link of the proper motions as demonstrated by Odenkirchen \& Brosche (1995).

The second method uses stars measured on plates of the Lick astrograph partly from the Lick Northern Proper Motion (NPM) program (Klemola et al. 1987) as described in detail in Geffert et al. (1997). 16 (NGC 4147) to 85 (NGC 7089) stars were used to calibrate our proper motions to an extragalactic reference frame by the determination of a constant offset of our proper motions with respect to the ones obtained from the Lick plates. The rms of the differences were 5 mas/a for each field, which is in good agreement with the quoted errors for the NPM program (Klemola et al. 1987). Four of our fields were calibrated by this method, which is denoted by LICK in Col. 5 of Table 1. According to (Brosche et al. 1991b) the zero 
Table 1. The fields for the calibration of the Hipparcos proper motion system. Column 5 denotes the method of the extragalactic calibration of the proper motions (see Sect. 4)

\begin{tabular}{lrrrrrrr}
\hline Field & $\begin{array}{r}\alpha_{2000} \\
{[\mathrm{hhmmss}]}\end{array}$ & $\begin{array}{r}\delta_{2000} \\
{[\mathrm{ggmmss}]}\end{array}$ & NS & $\begin{array}{r}\text { Link method } \\
\text { (see chapter 4) }\end{array}$ & Epoch intervall & Number of plates & Reduction with H37 \\
\hline P0735+178 & 073807 & +174219 & 6 & EO & $1900-1983$ & 11 & no \\
OJ 287 & 085449 & +200632 & 6 & EO & $1902-1980$ & 15 & yes \\
NGC 4147 & 121006 & +183231 & 9 & LICK & $1917-1979$ & 18 & yes \\
3C 273 & 122907 & +020309 & 4 & EO & $1910-1986$ & 15 & no \\
OQ 208 & 140700 & +282715 & 6 & LICK + EO & $1915-1994$ & 12 & yes \\
NGC 6218 & 164715 & -015652 & 6 & LICK & $1902-1988$ & yes \\
3C 371 & 180651 & +694928 & 4 & EO & $1893-1979$ & no \\
3C 390.3 & 184209 & +794617 & 3 & EO & $1894-1985$ & 12 & no \\
NGC 7089 & 213329 & -004923 & 4 & LICK + S & $1902-1988$ & yes \\
\hline
\end{tabular}

point uncertainty of this link method is of the order of $1.3 \mathrm{mas} / \mathrm{a}$.

For the third method galaxies and stars were measured on Palomar glass copies and ESO Schmidt plates in the same manner as done by Geffert et al. (1993) for the calibration of proper motions in the field of M 15. This method was applied only in the NGC 7089 field. The zero point accuracy of the proper motions is of the order of $1.3 \mathrm{mas} / \mathrm{a}$. In the field of OQ 208 and NGC 7089 we were able to perform two independent extragalactic calibrations of the proper motions. It is very satisfying to note that in both fields the results of both methods agree on the level of 2 mas/a.

\section{Absolute proper motions for 48 stars}

Table 2 gives positions and proper motions of 48 stars from our measurements. As mentioned above the system of the proper motions is defined in each field independently by the link procedure. Although these data were mainly provided for the calibration of the Hipparcos proper motions, they may be also used for the calibration of any astrometric catalogues, especially also for future satellite projects like DIVA (Bastian et al. 1996) and GAIA (Lindegren et al. 1994). All of our data were provided to the working group on the extragalactic link of the Hipparcos system. Besides the fact that a complete discussion is given in the papers by Tucholke et al. (1997) and Kovalevsky et al. (1997) a rough estimate of the accuracy of our data is given as follows.

We have compared our final proper motions with the corresponding ones from the H37 catalogue. We obtained a rms of the differences of 3 mas/a, which in the worst case may be taken as an upper limit of the accuracy of our data. For this comparison we were able to use data of 47 common stars. Due to a possible rotation of the H37 system with respect to our data, a complete comparison has to include a rotation of the two systems. We have applied a program given by Sinachopoulos (1986), which
Table 3. Mean estimated uncertainty of our proper motions determined by the comparison with H37 stars

\begin{tabular}{lrc}
\hline Method of comparison & $\begin{array}{r}\sigma_{\mu} \\
{[\mathrm{mas} / \mathrm{a}]}\end{array}$ & $\mathrm{NS}$ \\
\hline direct comparison & 3.0 & 47 \\
$\begin{array}{l}\text { with rotation } \\
\begin{array}{l}\text { with rotation and } \\
\text { excluding 6 outlayers }\end{array}\end{array}$ & 2.8 & 47 \\
$\begin{array}{l}\text { only fields } \\
\text { reduced to H37 }\end{array}$ & 2.3 & 31 \\
\hline
\end{tabular}

NS is the number of stars.

has been used before for the determination of systematic differences between radio and optical positions (Brosche \& Sinachopoulos 1987). A modification of that program was also the base for the calculation of the rotation of the Hipparcos data for all Bonn fields. Since the rotation angles of the solutions are discussed elsewhere we will restrict ourselves here to the accuracy of our data, which may be estimated by the rms of the deviations. The rms of the comparison including a possible rotation was $2.8 \mathrm{mas} / \mathrm{a}$ for all $47 \mathrm{stars}$. This result improves to $2.5 \mathrm{mas} / \mathrm{a}$ by omitting 6 outlying stars on the $3 \sigma$ level. Taking only the fields which had been reduced to the H37 catalogue improves the result slightly to $2.3 \mathrm{mas} / \mathrm{a}$. Table 3 gives the results of our comparison. Since we expect that the accuracy of our data will be mainly responsible for these deviations, Table 3 gives mainly the uncertainty of our proper motions. 
Table 2. 48 stars with absolute proper motion for the calibration of the Hipparcos proper motion system. The epoch of the observations is 1950. The internal accuracy of the proper motions is of the order of 2 mas/a

\begin{tabular}{|c|c|c|c|c|c|}
\hline Designation & HIC number & $\begin{array}{r}\alpha_{2000} \\
{[\text { hhmmss.sss }]}\end{array}$ & $\begin{array}{r}\delta_{2000} \\
{[\mathrm{ggmmss} . \mathrm{ss}]}\end{array}$ & $\begin{array}{l}\mu_{\alpha} \cos \delta \\
{[\mathrm{mas} / \mathrm{a}]}\end{array}$ & $\begin{array}{r}\mu_{\delta} \\
{[\mathrm{mas} / \mathrm{a}]}\end{array}$ \\
\hline $\mathrm{BD}+171617$ & 36921 & 073524.484 & +172259.80 & -11.6 & -11.1 \\
\hline $\mathrm{BD}+181681$ & 37009 & 073624.680 & +181055.98 & -16.0 & -25.9 \\
\hline $\mathrm{BD}+171623$ & 37074 & 073705.758 & +165415.45 & -1.8 & -6.9 \\
\hline $\mathrm{BD}+181692$ & 37160 & 073811.914 & +175028.53 & +12.9 & -6.2 \\
\hline $\mathrm{BD}+181701$ & 37300 & 073928.586 & +174028.33 & +1.8 & -7.4 \\
\hline $\mathrm{BD}+171634$ & 37436 & 074105.828 & +165223.89 & -6.4 & +8.9 \\
\hline G $9-29$ & 43552 & 085210.477 & +200843.80 & +191.8 & -152.3 \\
\hline $\mathrm{BD}+192119$ & 43642 & 085320.688 & +191920.42 & -22.9 & -15.6 \\
\hline $\mathrm{BD}+202232$ & 43683 & 085355.414 & +195802.05 & -23.2 & -10.6 \\
\hline $\mathrm{BD}+202233$ & 43701 & 085400.695 & +201349.63 & -54.1 & -7.9 \\
\hline $\mathrm{BD}+211939$ & 43730 & 085421.594 & +203510.55 & -21.9 & -8.4 \\
\hline $\mathrm{BD}+202244$ & 43935 & 085657.156 & +201159.97 & +14.7 & -13.2 \\
\hline $\mathrm{BD}+202683$ & 59121 & 120722.977 & +192111.98 & +13.3 & -2.1 \\
\hline $\mathrm{BD}+192531$ & 59152 & 120750.492 & +185659.42 & +119.7 & -70.3 \\
\hline $\mathrm{BD}+192533$ & 59234 & 120855.609 & +181813.91 & -15.1 & +19.1 \\
\hline $\mathrm{BD}+192534$ & 59238 & 120902.383 & +181920.41 & -121.7 & -61.2 \\
\hline $\mathrm{BD}+192536$ & 59310 & 121005.008 & +185838.25 & -159.6 & -37.1 \\
\hline $\mathrm{BD}+192539$ & 59391 & 121102.336 & +183731.00 & -40.8 & -20.6 \\
\hline $\mathrm{BD}+182676$ & 59418 & 121118.773 & +175844.17 & +3.1 & +18.1 \\
\hline $\mathrm{BD}+192541$ & 59550 & 121246.570 & +183203.55 & +86.7 & -26.3 \\
\hline $\mathrm{BD}+192542$ & 59571 & 121257.367 & +185828.64 & -19.5 & +1.6 \\
\hline $\mathrm{BD}+022539$ & 60667 & 122600.961 & +020231.02 & -39.1 & -2.8 \\
\hline $\mathrm{BD}+032644$ & 60713 & 122633.258 & +022632.56 & +5.7 & -7.8 \\
\hline $\mathrm{BD}+012701$ & 60902 & 122854.531 & +010900.07 & -99.5 & -101.0 \\
\hline $\mathrm{BD}+032660$ & 61096 & 123117.406 & +021809.05 & -14.1 & +1.2 \\
\hline $\mathrm{BD}+292486$ & 68765 & 140427.594 & +290834.72 & +7.4 & -32.2 \\
\hline $\mathrm{BD}+292488$ & 68793 & 140453.750 & +290221.91 & +7.5 & -3.9 \\
\hline $\mathrm{BD}+282292$ & 68903 & 140625.578 & +274333.78 & -40.3 & +33.0 \\
\hline $\mathrm{BD}+292491$ & 68928 & 140640.359 & +282903.16 & -5.9 & -17.2 \\
\hline $\mathrm{BD}+292493$ & 68955 & 140656.094 & +282617.00 & -69.7 & +4.0 \\
\hline $\mathrm{BD}+292497$ & 69024 & 140749.906 & +283243.53 & +23.1 & -7.8 \\
\hline $\mathrm{BD}-013242$ & 82040 & 164531.203 & -013441.51 & -9.9 & -3.2 \\
\hline $\mathrm{BD}-013243$ & 82070 & 164549.250 & -011551.11 & -20.4 & +8.8 \\
\hline $\mathrm{BD}-013244$ & 82114 & 164624.438 & -012134.22 & +40.8 & -76.1 \\
\hline $\mathrm{BD}-024247$ & 82152 & 164659.734 & -022912.25 & -5.5 & -11.4 \\
\hline $\mathrm{BD}-013251$ & 82274 & 164840.359 & -015209.98 & -0.1 & -0.3 \\
\hline $\mathrm{BD}-013254$ & 82382 & 165003.203 & -020205.51 & -8.9 & -12.9 \\
\hline $\mathrm{BD}+70973$ & 88295 & 180150.172 & +701418.31 & -12.8 & -22.3 \\
\hline $\mathrm{BD}+69968$ & 89005 & 180955.766 & +694040.13 & -25.1 & +198.5 \\
\hline $\mathrm{BD}+69970$ & 89113 & 181114.984 & +691456.31 & +14.7 & +38.4 \\
\hline $\mathrm{BD}+69973$ & 89571 & 181641.063 & +694234.88 & -37.8 & +1.2 \\
\hline $\mathrm{BD}+79604$ & 92040 & 184537.797 & +795630.50 & +18.2 & +71.6 \\
\hline $\mathrm{BD}+79605$ & 92208 & 184732.391 & +800934.50 & +18.4 & +81.1 \\
\hline $\mathrm{BD}+79610$ & 93162 & 185834.469 & +792320.25 & +7.7 & +13.7 \\
\hline $\mathrm{BD}-014172$ & 106324 & 213205.016 & -004254.76 & +18.9 & +6.0 \\
\hline $\mathrm{BD}-014173$ & 106385 & 213249.516 & -005258.80 & -6.1 & -7.4 \\
\hline $\mathrm{BD}-014177$ & 106603 & 213525.734 & -005853.37 & +28.0 & +21.7 \\
\hline $\mathrm{BD}-014178$ & 106655 & 213610.766 & -011816.96 & +22.5 & -30.2 \\
\hline
\end{tabular}




\section{Conclusions}

We have presented absolute proper motions of 48 Hipparcos from measurements of photographic plates. These data have been included in the calibration of the Hipparcos proper motion system (Kovalevsky et al. 1997). The accuracy of one single proper motion is of the order of 2 to $3 \mathrm{mas} / \mathrm{a}$.

Acknowledgements. We are indebted to many staff members of the observatories of Bordeaux, Bruxelles, Heidelberg, Leiden, RGO and Vienna for continuous support, which enabled us to use their plates. We thank A. Karafistan, C. Ducourant for the measurements of some of the plates and D. Sinachopoulos for providing us the program used in Sect. 4.

\section{References}

Bastian U., Röser S., Høg E., et al., 1996, AN 317, 281

Brosche P., 1980, AJ 85, 1674

Brosche P., Geffert M., 1981, A\&A 103, 78

Brosche P., Sinachopoulos D., 1987, MNRAS 227, 341

Brosche P., Ducourant C., Galas R., Geffert M., Karafistan A., 1991a, A\&A 245, 669

Brosche P., Tucholke H.-J., Klemola A.R., et al., 1991b, AJ 102, 2022

Dick W.R., Tucholke H.-J., Brosche P., et al., 1993, A\&A 279,
267

Froeschlé M., Kovalevsky J., 1982, A\&A 116, 89

Geffert M., 1987, Absolute proper motions of globular clusters. in: Azzopardi M. and Matteucci F. (eds.), Stellar evolution in the Outer Halo of the Milky Way, ESO Conference Workshop Proceedings, München, p. 351

Geffert M., Tucholke H.-J., Walter H.G., et al., 1989, A\&A 224,323

Geffert M., Colin J., LeCampion J.-F., Odenkirchen M., 1993, AJ 105, 168

Geffert M., Krümmel M., Schmidt H., 1995, A\&AS 112, 229

Geffert M., Klemola A.R., Hiesgen M., 1997, A\&AS (submitted)

Klemola A.R., Jones B.F., Hanson R.B., 1987, AJ 94, 501

Kovalevsky J., Lindegren L., Johnston K.J., et al., 1997, A\&A (in press)

Lindegren L., Perryman M.A.C., Bastian U., et al., 1994, GAIA - Global Astrometric Interferometer for Astrophysics. in: Breckenridge J.B. (ed.): Amplitude and Intensity Spatial Interferometry II, SPIE Conf. Proc. 1947, p. 147

LeCampion J.-F., Geffert M., Dulou M.-R., Colin J., 1992, A\&AS 95, 233

Odenkirchen M., Brosche P., 1995, A\&A 302, 915

Sinachopoulos D., 1986 (private communication)

Tucholke H.-J., Brosche P., Odenkirchen M., 1997, A\&AS (in press) 